

\title{
Pucho Vs. Vapo - Smoking versus vaping
}

\author{
Ezequiel Arrieta, Facundo Mónaco
}

Ilustradora: Mariana Ruiz Johnson

Fecha de publicación: 30/5/20

Link a la nota original: https://elgatoylacaja.com/pucho-vs-vapo/

\section{Abstract}

Humans have been consuming tobacco for 4500 years. It started as a part of religious rituals but became popular when suggested to cure diseases and considered cool. Nowadays, it is associated with $15 \%$ of total deaths worldwide, and represent one of the main Public Health issues in the agenda. Most tobacco users need between eight to ten attempts to quit smoking. In this context, vaping emerges as a safer alternative to conventional smoking. However, vaping might not be completely safe. In this article, originally published in El Gato y La Caja, pros and cons of vaping versus smoking are presented. 
Todos tenemos nuestro mundo de cosas que nos gustan y que, aunque sabemos que pueden hacernos mal, elegimos seguir consumiendo/haciendo. No hablamos solamente del alcohol, el tabaco, las grasas saturadas o los azúcares sino también, por ejemplo, tomar sol al mediodía, hacernos un arito en la nariz, meternos al mar cuando no hay guardavidas, tirarnos con paracaídas. Decisiones que tomamos a diario (y no tan), que somos conscientes de que implican cierto riesgo, que potencialmente pueden hacer daño a nuestra salud $y$, así y todo, elegimos hacerlo.

La humanidad consume tabaco desde hace al menos 4500 años, aunque no de la manera que hacemos ahora, sino asociada a ciertos rituales religiosos dentro de culturas particulares. La expansión del hábito tal cual lo conocemos hoy se puede rastrear en algún momento del siglo XVI, cuando el embajador francés Jean Nicot sugirió que las hojas del tabaco podían curar un montón de enfermedades. Desde ahí, el crecimiento en su consumo se mantuvo constante hasta hace relativamente poco y, tras una ola de cáncer y concientización, ha dejado de ser el accesorio cool que fue décadas atrás. Pero a pesar de las subas en el precio del atado, de las fotos de pies putrefactos o bebés corrompidos, y de tantos otros intentos por alejarnos de esos rollitos de tabaco, fumadores sigue habiendo por montones. Y frases como "Estoy tratando de dejar de fumar, esta es una opción menos dañina" las escuchamos últimamente en el laburo, en el club, en la juntada con amigos y en donde sea que haya una persona que esté intentando dejar el cigarrillo, o al menos bajar la cantidad diaria, a través de la utilización de vapeadores. Porque el vaping ganó su lugar.

'Vapear' es, en simples palabras, inhalar un vapor originado en un dispositivo (vaper, vaporizador o cigarrillo electrónico). Los cigarrillos electrónicos funcionan evaporando un líquido mediante el calor generado por una resistencia en su interior (alimentada por una batería) para luego ser inhalado por el usuario. Los líquidos utilizados para dicho fin son de composición diversa, aunque en líneas generales tienen como componente principal al propilenglicol o la glicerina (encargados de crear una sensación en la garganta similar a la de fumar), y suelen incluir saborizantes y aromatizantes (frutilla, chocolate, vainilla y otros). Además, el líquido puede contener nicotina y su presencia (o ausencia), así como su concentración, representan datos importantísimos para este debate.

En líneas generales, si lo comparamos con el cigarrillo tradicional, el vaper tiene algunas ventajas: además de ser una opción inodora, no deja aliento a 
cenicero, evita los agujeros en el mantel y en la ropa del que baila al lado, pueden consumirse saborizados sin nicotina (de hecho, por ejemplo en Australia, estos son los únicos legalmente autorizados para la venta) y aquellos líquidos que poseen nicotina no presentan niveles tan elevados (entre 0 y $24 \mathrm{mg} / \mathrm{ml}$, de hecho, por ejemplo en Estados Unidos, el más fuerte que puede conseguirse, por ley, tiene 20 $\mathrm{mg})$.

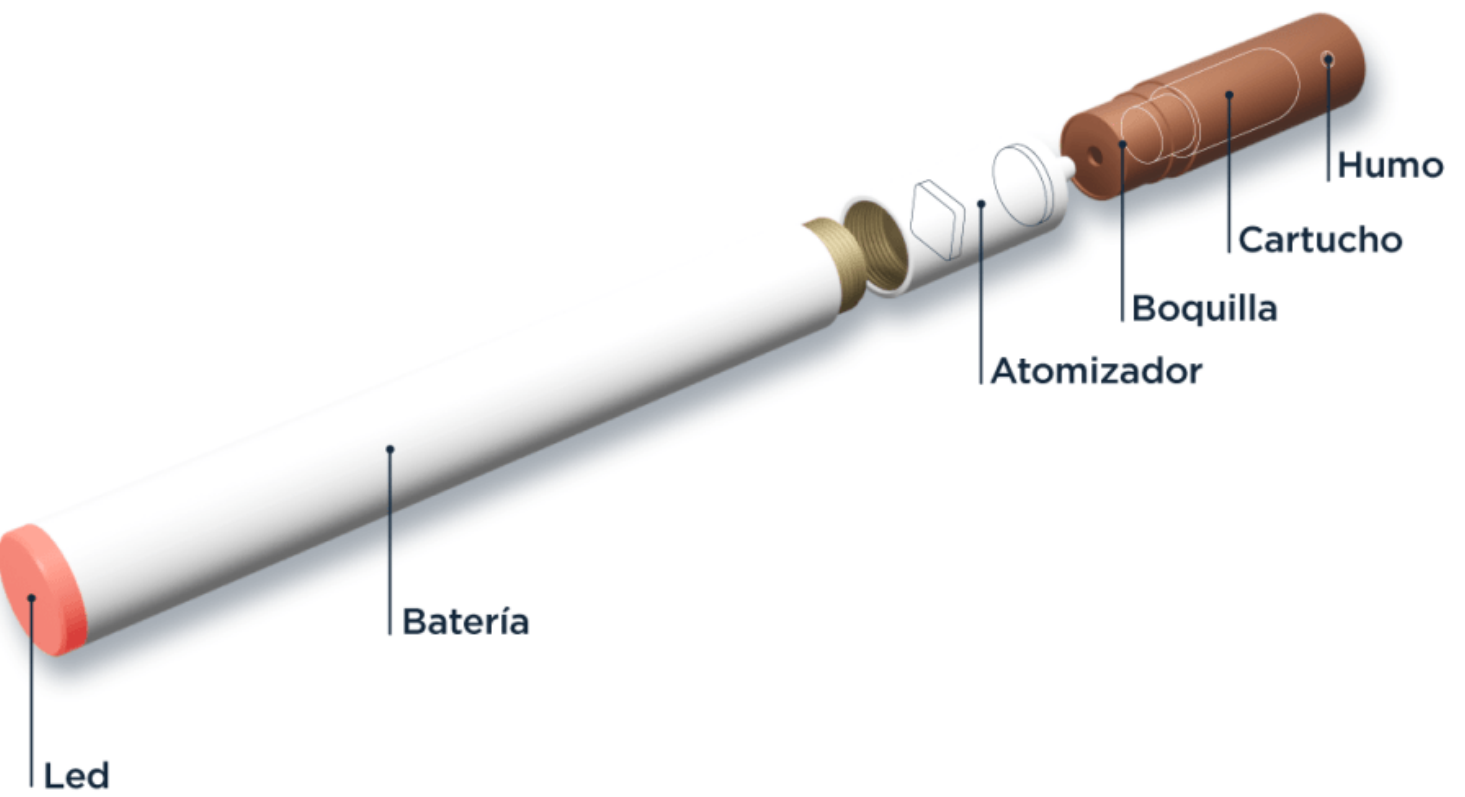

Fuente: Organización Mundial de la Salud

Entonces, a simple vista, el vapeador parece una opción poco nociva y bastante menos peor que el cigarrillo, pero ¿cuál es la posta? La respuesta corta es: depende de quien se trate.

\section{Cortina de humo}

Antes de seguir con el cigarrillo electrónico, hagamos un breve repaso por su contraparte: el cigarrillo tradicional. Ya no debería ser novedad para nadie que el pucho enferma, y mucho. Abordar profundamente los efectos en la salud del consumo de cigarrillos llevaría un libro en sí mismo, pero en líneas generales, fumar es un factor de riesgo importante para una gran cantidad de enfermedades y los efectos aparecen ni bien se empieza a consumir tabaco, aumentan con el paso de los años y la cantidad de 
cigarrillos fumados. Actualmente se lo considera uno de los mayores problemas de Salud Pública del mundo, dado que se asocia al 15\% de las muertes totales (superado sólo por la hipertensión arterial) y el $8 \%$ de los años de vida perdidos por discapacidad. En Argentina, las cosas son algo peores: el consumo de tabaco está asociado al $18 \%$ de las muertes y al $\mathbf{1 3} \%$ de los años de vida saludable perdidos por discapacidad. Todo esto genera elevados costos sanitarios que representan el $12 \%$ del monto que el país gasta en salud cada año, equivalente al $1 \%$ del Producto Bruto Interno. El sitio web del Programa Nacional de Control de Tabaco del Ministerio de Salud tiene muchos datos sobre los efectos a la salud del consumo de tabaco para quienes quieran profundizar. Nada que no sepamos por las bellas imágenes adjuntas al atado, bah.

\section{Tendencia de consumo de tabaco en adultos y adolescentes argentinos}

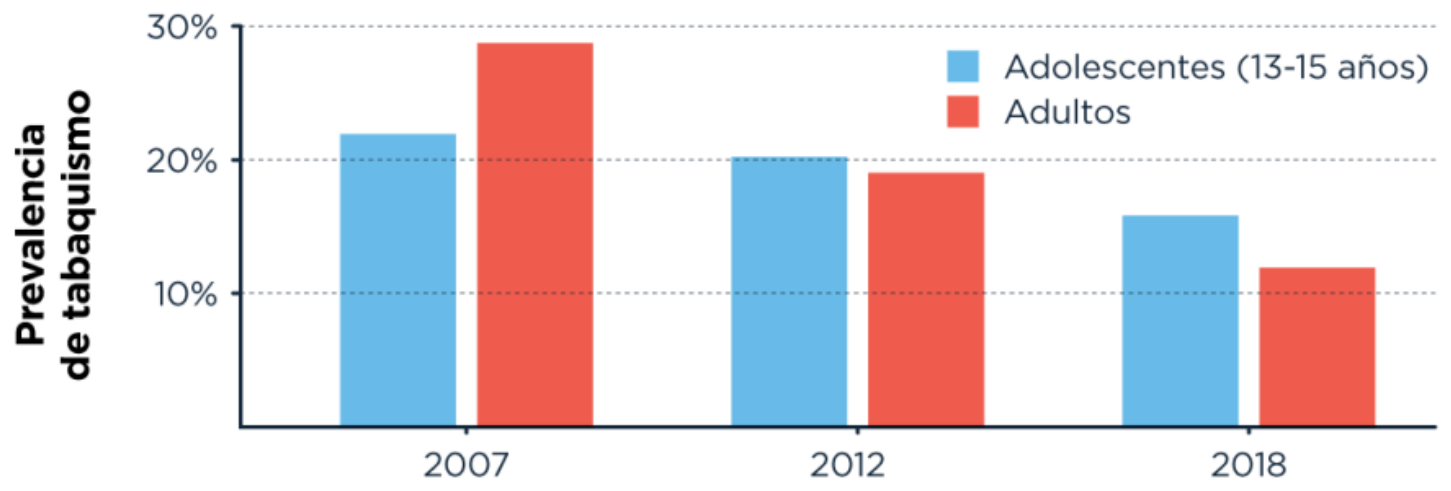

Año

Fuente: Encuestas Mundiales de la Salud Escolar y Encuestas Nacionales de Factores de Riesgo.

Afortunadamente, la prevalencia en el consumo de tabaco viene bajando en todo el mundo a un ritmo interesante tanto en adultos como en adolescentes. En Argentina, la reducción observada desde el año 2005 al año 2018 fue de un 24\%. Esta disminución en el consumo de tabaco se dio gracias al trabajo en equipo entre gobiernos, organismos internacionales y ONGs que ha permitido desarrollar e implementar políticas públicas orientadas a reducir las muertes por el consumo de tabaco y la exposición al humo del cigarrillo ajeno, como es el caso del Convenio Marco para el Control del Tabaco de la Organización Mundial de la Salud. Este tratado internacional ha sido ratificado por casi 180 países para combatir de manera global la epidemia del tabaquismo y dar una respuesta sanitaria internacional a las estrategias que utiliza la industria tabacalera para incentivar el consumo de cigarrillos. 
Pero más allá del reconocido lobby que ejerce la industria tabacalera sobre la implementación de las leyes anti-tabaco, tenemos un problema difícil de manejar: las personas disfrutan la experiencia de consumir tabaco y su potente capacidad adictiva las envuelve en un loop eterno de deseos de fumar. Unos 10 segundos después de haber dado una pitada, comienzan las sensaciones estimulantes mientras aumenta la relajación y se reduce el estrés (y la ansiedad). Así, en el contexto de la vida moderna, el cigarrillo se convirtió para muchos en un salvavidas de regulación emocional ante situaciones de ansiedad al generar relajación inmediata después de su consumo, aunque el alivio es solo temporario. Además, a diferencia del alcohol u otras sustancias psicoactivas, la nicotina altera la conciencia de maneras que posibilitan la concentración y el trabajo.

Si bien existen herramientas terapéuticas para el cese tabáquico, que van desde los psicofármacos hasta los chicles o parches con nicotina, el proceso no es para nada sencillo. Seguro que todos tienen un amigo de un amigo que dejó de fumar de un día para el otro porque así se lo propuso, pero la enorme mayoría de los usuarios necesitan de ocho a diez intentos para tener éxito. $Y$ eso puede llegar a ser muy frustrante, puede causar abatimiento y desmoralización que pueden conducir hacia un camino de entrega total al deterioro progresivo e irreversible de la salud. Las personas somos bichos complejos, y atribuir una única causa a la incapacidad para abandonar el consumo de una sustancia (falta de fe o tener una moral quebrada) habla más de los prejuicios del juez que de la realidad cognitiva del acusado. Hace casi 40 años que sabemos que la abstinencia no es una meta realista en un grupo considerable de adictos, al mismo tiempo que existen otros tantos que no están interesados en eliminar por completo el consumo debido a que disfrutan la actividad. Ante esta pared de realidad, deberíamos explorar soluciones alternativas al abstencionismo que nos permitan reducir los riesgos y daños de los usuarios, de los que están alrededor y al ambiente. Un camino con el menor sufrimiento posible.

\section{Mi cuerpo es un templo profano}

Como dijimos, el hábito de fumar tabaco es un factor importantísimo que predispone a la aparición de un montón de enfermedades y esto genera sufrimiento, muertes y gastos que serían evitables. ¿Qué sabemos sobre los efectos a la salud del vapo? La realidad es que no mucho. No hay una suficiente cantidad de estudios que nos permitan sacar conclusiones sólidas, pero nos animamos a decir que probablemente el vaping no esté libre de riesgos. Sin embargo, disponemos de algunos datos para reconocer un patrón de efectos que, por supuesto, se irá modificando a medida que se sumen estudios de diversa índole (in vitro, con animales humanos y no-humanos). 
https://www.youtube.com/watch?v=LOZuxwVk7TU\&feature=emb_logo

Britney y su video ensayo sobre consumos problemáticos.

En el humo del tabaco se han descrito más de 4000 sustancias químicas diferentes, muchas de ellas son potentes carcinógenos, como el formaldehído. En el caso de los vapers, un estudio reciente encontró que el calentamiento del propilenglicol también puede generar esta sustancia, pero dichos experimentos fueron realizados en condiciones irreales, utilizando modelos de vapers antiguos y con temperaturas muy por encima a las utilizadas por los usuarios, por lo que fueron ampliamente criticados por la comunidad científica. En el aerosol del vaporizador, al tener una composición diferente al tabaco del cigarrillo y al no haber combustión, la presencia y concentración de estas sustancias cancerígenas es muchísimo menor. Respecto a la nicotina, si bien esta sustancia es la responsable del potencial adictivo, su influencia sobre el desarrollo de un cáncer es mínima. De todas maneras, la aparición de un cáncer de pulmón necesita de unos 30 años desde la primera pitada, por lo que se necesitan más estudios a largo plazo para comprender mejor las implicancias del vaping sobre el cáncer más frecuente en los fumadores.

En cuanto a enfermedades cardiovasculares, de nuevo, no existen estudios a largo plazo que nos permitan hacer un análisis certero, pero nos animamos a decir que el vaping con nicotina no está libre de riesgos. Sabemos que la inhalación del vapor con nicotina aumenta la frecuencia cardíaca y la presión arterial, aunque los efectos son menores a los generados por el cigarrillo tradicional. A pesar de ello, la nicotina no deja de ser un factor de riesgo para el infarto de corazón y el accidente cerebrovascular. De todas maneras, el efecto depende de si la persona fumaba o no antes de usar el vaper. También se encontraron efectos negativos de la inhalación de vapor sobre el funcionamiento del endotelio, la capa interna de las arterias y venas que, cuando anda mal, es un punto de partida para el desarrollo de arteriosclerosis y sus consecuencias asociadas. Sin embargo, los efectos dañinos al sistema cardiovascular asociados al cigarrillo electrónico son menores a los de los cigarrillos tradicionales y el estado de los vasos sanguíneos es similar al de las personas no-fumadoras (probablemente porque se evitan las emisiones tóxicas propias de la combustión). No tenemos datos disponibles sobre efectos cardiovasculares a largo plazo o efectos en personas con enfermedad cardiovascular de base, pero el debate continúa. 
Por último, enfermedades respiratorias: a pesar de que el consumo oral del propilenglicol sea considerado seguro, la situación parece ser otra cuando se lo lleva a hacia la vía respiratoria. Aún no sabemos exactamente cómo impacta el vapor de propilenglicol y glicerina sobre los pulmones, pero hay evidencia de que su vaporización puede causar irritación de los ojos, garganta y vía respiratoria (lo cual no sería grave si la exposición es única, pero la cosa cambia cuando es repetitiva). Otra preocupación es la relacionada al impacto de los saborizantes y aromatizantes contenidos en el líquido, los cuales parecen inducir un proceso de inflamación, paso necesario para desarrollar una enfermedad pulmonar obstructiva crónica (EPOC), aunque esta respuesta inflamatoria parece ser menor que la causada por los cigarrillos convencionales.

En este estudio reciente se hizo un seguimiento durante 3 años a un grupo de adultos con el fin de evaluar el riesgo de desarrollar una enfermedad pulmonar crónica (EPOC, bronquitis crónica, enfisema y asma). El resultado fue que las probabilidades de desarrollar algunas de estas enfermedades fueron mayores para los fumadores de tabaco que para los usuarios de cigarrillos electrónicos, y aún mayores para los consumidores de ambos productos. Pero más allá de las enfermedades a largo plazo que pueda causar, el cigarrillo electrónico encendió la alarma y el debate público tras darse a conocer la aparición de casos de personas con dificultad respiratoria luego de vapear, algunos de los cuales terminaron en muerte. Si bien la 'lesión pulmonar asociada al uso de productos de cigarrillos electrónicos' es posible, lo cierto es que el $83 \%$ de las personas afectadas dijeron haber vapeado líquidos no diseñados para ser usados en el vaper (como el aceite de cannabis).

Por otro lado, al ser estructuras metálicas, es esperable la emisión de metales por parte del vaper. Se encontraron algunos metales en niveles más altos en comparación con los cigarrillos de tabaco pero, en general, los niveles que se detectaron están por debajo de los límites de seguridad para medicamentos inhalados o para exposición ocupacional, y no parecen representar un riesgo significativo para la salud. Respecto a la nicotina, si bien los cigarrillos electrónicos aportan menor cantidad de nicotina por vapeada cuando se los compara con la nicotina contenida en cada pitada de los cigarrillos convencionales, se puede vapear indefinidamente hasta que se acaba la batería. Por lo tanto, en función de la concentración de nicotina de cada cartucho, de su volumen total y del número de vapeos, teóricamente se podría llegar a alcanzar un alto contenido de nicotina en sangre de forma menos controlable y tóxica. Pero es poco probable que un usuario llegue a hacer un fondo blanco de nicotina hasta el desmayo. 


\section{Daños asociados a diferentes formas de consumir nicotina}

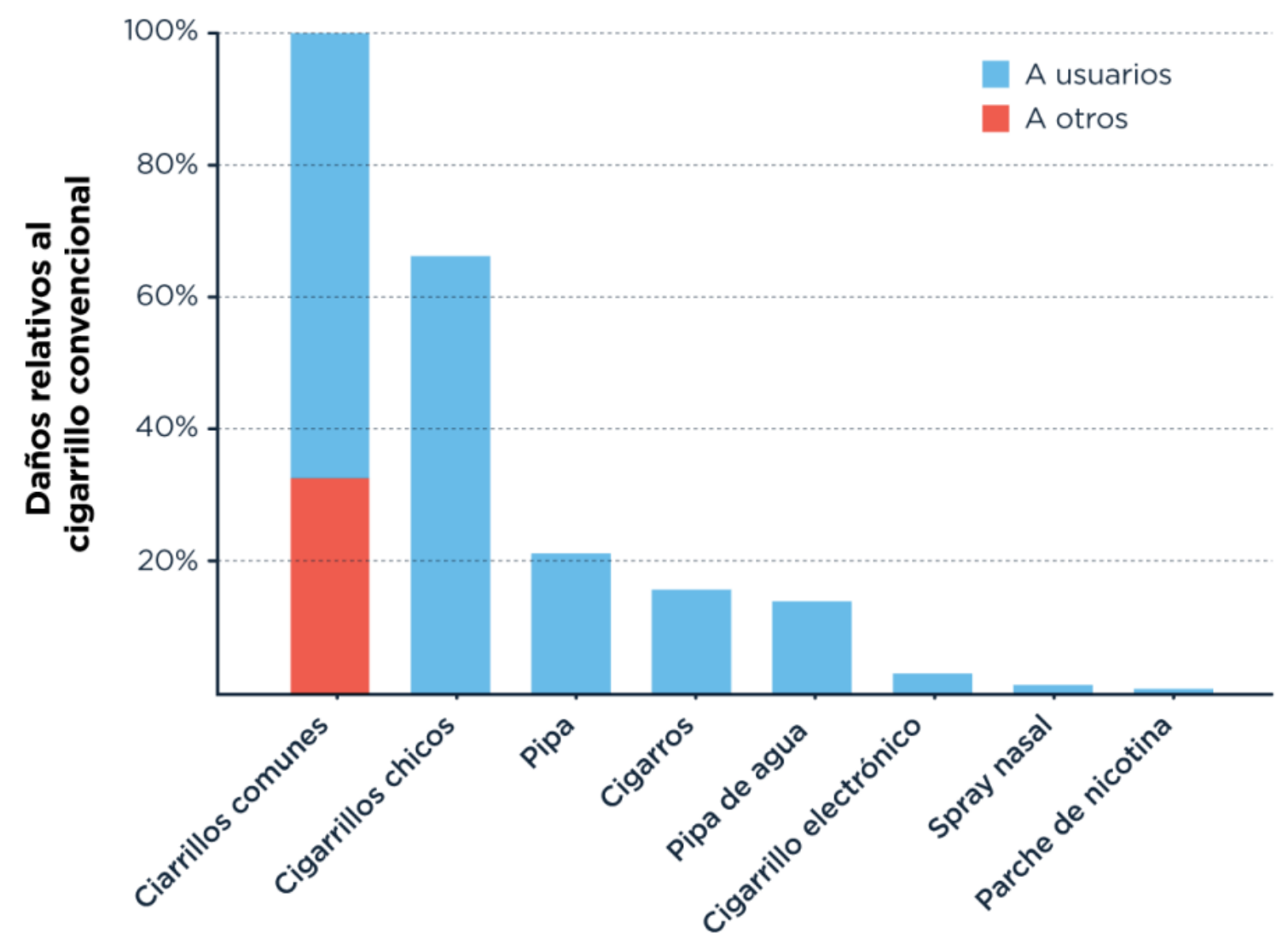

Valores relativizados al daño por cigarrillos. Cuando se compara al cigarrillo electrónico con el cigarrillo convencional y otros productos que permiten el consumo de nicotina (como pipas y bongs), los vapers producen considerablemente menos daño al usuario. Fuente.

\section{Radiopasillo industrial}

Ante la enorme carga de salud que representa el consumo de cigarrillos y el gran desafío sanitario que implica reducir su consumo, es necesario reflexionar sobre todas las opciones posibles con el objetivo de (al menos) disminuir los daños y las muertes asociadas a este real flagelo. Los cigarrillos electrónicos no sólo son capaces de reducir los daños en aquellas personas que no quieren abandonar el consumo de nicotina, sino que además han mostrado ser una excelente opción para el cese tabáquico, incluso con mejor resultado que los parches de nicotina. A pesar de ello, estos dispositivos son ampliamente demonizados y su acceso limitado. Por lo pronto, en Argentina el 
cigarrillo electrónico es ilegal y está prohibido por la ANMAT (Administración Nacional de Medicamentos, Alimentos y Tecnología Médica).

Además, los rumores alimentados por el tan sobrevalorado sentido común no ayudan a mejorar la situación: se cree que los vaporizadores son impulsados por la industria del tabaco para generar nuevos adictos que después saltan al pucho, una especie de puerta de entrada hacia la droga más peligrosa que conocemos. Pero los estudios demuestran que no existen tal salto, y que los nuevos consumidores (los de vaporizador) en realidad lo que menos quieren es consumir nicotina, sino más bien vapores con sabor a frutilla, vainilla o menta. Por supuesto, no es deseable que los no-fumadores comiencen a usar cigarrillos electrónicos y se debería desalentar el uso en esta población, particularmente en los adolescentes. Pero en el caso de los fumadores (el $20 \%$ de la población nacional), el reemplazo total o parcial del cigarrillo convencional por el electrónico constituye una clara ganancia para la salud individual y pública que deberían contemplarse seriamente.

Lo mismo ocurre en la población adolescente que tanto nos preocupa que utilicen los vapers: el vapeo suele ser experimental y no persiste en el tiempo, y los adolescentes que mantienen el uso del cigarrillo electrónico son aquellos que fumaban cigarrillos convencionales. Siempre es bueno ocuparnos y preocuparnos por los más jóvenes, pero tenemos evidencias contundentes que nos dicen que la prohibición y la difusión de información falsa no nos ayudará a frenar el consumo de sustancias psicoactivas en los adolescentes. La educación tradicional que reciben sobre el uso de sustancias psicoactivas se basa más en el temor que en tener una discusión abierta y honesta, porque se cree que hablar sobre el tema los condenará a incursionar en conductas inmorales. Si a eso le sumamos la sobrestimación de los daños que provocan ciertas sustancias y el alarmismo generalizado, aumentan las probabilidades de debilitar un mensaje cuyo objetivo es informar y no infundir miedo.

\section{A lo hecho, pecho}

A pesar de que haya pocas dudas a la hora de determinar que los vapos son menos dañinos que los cigarrillos, estamos de acuerdo en que no son inofensivos y que no existe un consumo libre de riesgos. En cualquier etapa de la vida, el mejor consejo resulta 'siempre será más saludable no vapear, así que si no fumás, no te pegues al vaping'.

Sin embargo, existe una importante proporción de personas que son fumadoras de cigarrillos convencionales y que, por diversos motivos, no pueden o no quieren abandonar dicho consumo. Por supuesto, la mejor recomendación hacia ellos siempre será que dejen de fumar, y que para hacerlo, mejor recurrir a un profesional de la salud que utilice método basados en evidencia científica. Pero en lugar de acotar las opciones a "dejá de fumar o 
morite", como sociedad nos merecemos debatir y reflexionar sobre la posibilidad de incluir al cigarrillo electrónico como una herramienta terapéutica más en la resolución de este enorme problema de salud pública que tenemos entre manos. Para las personas que fuman y que no pueden o no quieren dejar de hacerlo, su mejor opción probablemente sea el cigarrillo electrónico.

Quizás, en este caso, optar por el mal menor no representa una actitud derrotista ante una realidad que no podemos cambiar, sino que implica abrazar a la diversidad de conciencias y contextos, e implementar abordajes integrales que contemplen la complejidad del problema. 


\section{Referencias}

Farsalinos K. (2018). Electronic cigarettes: an aid in smoking cessation, or a new health hazard?. Therapeutic Advances in Respiratory Disease, 12, 1753465817744960. doi:10.1177/1753465817744960

Muthumalage T, Prinz M, Ansah KO, Gerloff J, Sundar IK and Rahman I (2018) Inflammatory and Oxidative Responses Induced by Exposure to Commonly Used e-Cigarette Flavoring Chemicals and Flavored e-Liquids without Nicotine. Front. Physiol. 8:1130. doi: 10.3389/fphys.2017.01130

Dharma N. Bhatta, Stanton A. Glantz. (2019). Association of E-Cigarette Use With Respiratory Disease Among Adults: A Longitudinal Analysis. American Journal Of Preventive Medicine. 58(2), 182-190

Christiani DC. Vaping-Induced Acute Lung Injury. N Engl J Med. 2020;382(10):960-962. doi:10.1056/NEJMe1912032

Benowitz, Neal L, and Andrea D Burbank. "Cardiovascular toxicity of nicotine: Implications for electronic cigarette use." Trends in cardiovascular medicine vol. 26,6 (2016): 515-23. doi:10.1016/j.tcm.2016.03.001

Franzen, Klaas \& Willig, Johannes \& Talavera, Silja \& Meusel, Moritz \& Sayk, Friedhelm \& Reppel, Michael \& Dalhoff, Klaus \& Mortensen, Kai \& Droemann, Daniel. (2018). E-cigarettes and cigarettes worsen peripheral and central hemodynamics as well as arterial stiffness: A randomized, double-blinded pilot study. Vascular Medicine. 23. $1358863 \times 1877969$. $10.1177 / 1358863 \times 18779694$

Argentina. Ministerio de Salud de la Nación Guía rápida e-cig. - 1a ed. - Ciudad Autónoma de Buenos Aires : Ministerio de Salud de la Nación, 2017. 14 p. ; 22 × $15 \mathrm{~cm}$. ISBN 978-950-38-0247-2 1. Acceso a la Salud. 2. Neumonología. I. Título. CDD 614.592 